\title{
STATISTICS OF KLEIN POLYHEDRA AND MULTI-DIMENSIONAL CONTINUED FRACTIONS
}

\author{
M.L. Kontsevich $\left.{ }^{*, \dagger}\right)$ and Yu.M. Suhov ${ }^{\dagger, \star)}$
}

\begin{abstract}
A (generalized) Klein polyhedron (KP) is defined as the convex hull of a set of lattice points inside a (convex) domain $\mathbb{D} \subset \mathbb{R}^{n}$. We focus on two cases where $\mathbb{D}$ is the interior (i) of a simplicial cone or (ii) of a paraboloid. We introduce a dynamical system, with a group action and an invariant measure, which is naturally associated with the KP, and discuss statistical properties of the boundary of a (random) KP, with respect to the invariant measure. The boundary of a $\mathrm{KP}$ is called a veil; it is formed by 'faces' that are convex polyhedra of dimension $\leq n-1$. In the case of a simplicial cone, the dynamical systems constructed are multi-dimensional generalizations of the well-known Gauss automorphism (related to the continued fraction representation of a number $x \in(0,1))$. The case of a paraboloid is important as it emerges as the limiting case for the domains in $\mathbb{R}^{n}$ of the form $\lambda \mathbb{D}$, as $\lambda \rightarrow+\infty$, where $\mathbb{D}$ is a given convex domain with a smooth boundary. In particular, we are interested in the distribution of various parameters characterising local combinatorial and geometric properties of a veil, such as the number of lattice points lying on a single face, the number of vertices of a face, etc. We find that the two cases under consideration (simplicial and parabolic) are quite different from this point of view. A by-product of our approach is that in the simplicial case it allows us to propose algorithms for searching a 'good' rational approximation for the components of a given vector $\theta$; a particular algorithm of this kind was proposed earlier in [La].
\end{abstract}

\section{INTRODUCTION: THE GAUSS AUTOMORPHISM}

This paper was conceived as an attempt to generalize certain aspects of the theory of continued fractions to the multi-dimensional case; an initial motivation was to provide an answer to a question proposed by V.I. Arnol'd at his seminar at Ècole

Research supported in part by I.H.E.S., Bures-sur-Yvette, EC Grant 'Training Mobility and Research (Contract ERBMRXT-CT 960075A) and INTAS Grant 'Mathematical Methods for Stochastic Discrete Event Systems' (INTAS 93-820)

*) I.H.E.S., Bur-sur-Yvette, F-91440, France

$\dagger$ †) Institute for Problems of Information Transmission, Russian AS, Moscow 101447,

Russia

*) Statistical Laboratory, DPMMS, University of Cambridge, Cambridge CB2 1SB, and St John's College, Cambridge CB2 1TP, UK 
Normale Supèrieure, Paris, in the Spring of 1997. However, in the process of writing it, we decided to extend our considerations in other directions offered by the emerging approach. This approach may in particular be applied to the problem of asymptotical distribution of the number of integer points along the boundary of a domain $\partial(\lambda \mathbb{D})$ as $\lambda \rightarrow \infty$.

The continued fraction theory which was our original incentive is indeed a popular subject, both theoretically and with regard to numerous applications. Attempts to develop a multi-dimensional analogue were inspired in particular by the need to design algorithms that could find rational approximations for vectors, and integer ones for linear manifolds, in $\mathbb{R}^{n}$. See, e.g., [Br], [La] and references therein.

The continued fraction representation of a number $y \in(0,1)$ :

$$
y=\left(a_{0}+\left(a_{1}+\left(a_{2}+\left(a_{3}+\ldots\right)^{-1}\right)^{-1}\right)^{-1}\right)^{-1}, a_{i} \in \mathbb{Z}_{+}:=\{1,2, \ldots,\},
$$

associates with $y$ a sequence of positive integers $\left(a_{0}, a_{1}, \ldots\right)$ (accordingly, we will write $\left.y \sim\left(a_{0}, a_{1}, \ldots\right)\right)$. An important role in the 'one-dimensional' theory of continued fractions is played by the Gauss automorphism

$$
\gamma:\left(x_{1}, x_{2}\right) \in \mathbb{Q} \mapsto\left(\left\{\frac{1}{x_{1}}\right\}, \frac{1}{\left[x_{1}^{-1}\right]+x_{2}}\right),
$$

with the invariant probability measure

$$
\mu\left(\mathrm{d} x_{1} \times \mathrm{d} x_{2}\right)=\frac{\mathrm{d} x_{1} \mathrm{~d} x_{2}}{\left(1+x_{1} x_{2}\right)^{2}} \frac{1}{\ln 2}
$$

on the unit square $\mathbb{Q}=(0,1) \times(0,1)$; see, e.g., $[\mathrm{CFS}$. Here, and below, $[x]$ and $\{x\}=$ $x-[x]$ denote, respectively, the integer and fractional parts of a positive real number $x$. More precisely, if $x_{1} \sim\left(a_{0}, a_{1}, \ldots\right)$, and $x_{2} \sim\left(a_{-1}, a_{-2}, \ldots\right)$, then $\gamma\left(x_{1}, x_{2}\right)=\left(x_{1}^{\prime}, x_{2}^{\prime}\right)$ where $x_{1}^{\prime} \sim\left(a_{1}, a_{2}, \ldots\right)$ and $x_{2}^{\prime} \sim\left(a_{0}, a_{-1}, \ldots\right)$. In particular, the distribution of an entry $a_{i}$ in the continued fraction representation of numbers $x_{j} \in(0,1), j=1,2$, is given by

$$
\rho_{s}^{0}=\mu\left(a_{i}=s\right)=\log _{2} \frac{(s+1)^{2}}{s(s+2)}, s \in \mathbb{Z}_{+},
$$

whereas the expected value of $a_{i}$ is

$$
E^{0}=\sum_{s} s \rho_{s}^{0}=\int_{\mathbb{Q}} \mu\left(\mathrm{d} x_{1} \times \mathrm{d} x_{2}\right)\left[x_{1}^{-1}\right]=\int_{\mathbb{Q}} \mu\left(\mathrm{d} x_{1} \times \mathrm{d} x_{2}\right)\left[x_{2}^{-1}\right]=\infty
$$

(the meaning of the superscript ${ }^{0}$ will become clear later).

Recently, following [Ar 1,2], beautiful results were obtained in a series of papers starting with $[\mathrm{K}]$ (see also $[\mathrm{L}]$ and references therein), based on a geometric approach to the multi-dimensional continued fraction theory. A basic notion here is that of a 
Klein polyhedron (KP) inside a simplicial cone in $\mathbb{R}^{n}, n \geq 2$, with the corner at the origin. A KP is defined as the convex hull of the set of the lattice points inside the cone (excluding the origin). The boundary of such polyhedron may be considered as an $(n-1)-$ dimensional continued fraction; for $d=2$ the standard theory is recovered. Following popular terminology, we call this boundary a simplicial veil (or simply a veil ${ }^{1}$ ). So far, the main attention in this approach has been focused on the analysis of an individual veil, rather than that of its 'statistical' properties. In contrast, this paper (together with subsequent ones) aims to establish basic facts about statistical behaviour of the veil; we believe that such an approach may be of use in several applications, including the above-mentioned continued fraction algorithms and a popular problem of evaluating the number of integer points in a geometric locus (see, e.g., [CdV 1,2], [DRS] and references therein).

More precisely, the space of veils is endowed with a natural measure that is invariant under a group action. This leads us to a dynamical system (a veil flow) where the role of time is played by an appropriate subgroup of $\mathrm{SL}(2, \mathbb{R})$. One of the results of this paper, Theorem 1 (see Section 3 below), asserts that, with respect to the invariant probability measure, the expected number of lattice points on a 'typical', or 'average', face of a simplicial veil is infinite. (A face of a veil is itself a compact convex polyhedron of dimension $n-1$.) The above result is a direct generalisation of the statement that the expected value of a 'typical' entry of a continued fraction is infinite. In contrast, the expected number of lattice points lying on the boundary of a face (which is a union of compact convex polyhedra of dimension $n-2$ ) is finite. In general, the distribution of the number of lattice points on the lower-dimensional parts of the boundary of a face has several finite moments. Moreover, the distribution of the number of vertices of a face has a rapidly decaying tail: the probability that a face has $s$ vertices is $\sim \exp \left(-s^{1 /(n-2)}\right)$; see Theorem 3 in Section 3. The next problem is to identify the distribution of these random variables. So far we have not been able to find concise formulas, but that does not mean that they do not exist.

In principle, the same approach may be used to study any local combinatorial parameter of a veil: degrees of vertices of a face, the integer lengths of the edges, etc.

A similar dynamical system may be defined when a simplicial cone is replaced by a paraboloid in $\mathbb{R}^{n}$. Here, as before, a notion of a veil can be introduced (we use the term parabolic veils, to distinguish them from the simplicial ones). Properties of the dynamical system related to parabolic veils differ from those of the simplicial veil flow; in particular, the expected number of integer points on a face of a parabolic veil is finite; see Theorem 5 in Section 4. The particular role of parabolic veils is that they emerge as limits for veils associated with a general convex domain $\mathbb{D}$, in the course of 'inflating', or scaling, domain $\mathbb{D}$ by factor $\lambda$ and letting $\lambda \rightarrow \infty$. See Theorems 9-11 in Section 4 .

The rest of the paper is organised as follows. We begin with a revision of the Gauss automorphism (Section 2) and continue with results about simplicial veils (Section 3).

${ }^{1}$ The veil, besides its original meaning, is a synonym for sail in French, one of the working languages in this area. 
Section 4 contains results on parabolic veils. In Section 5 we discuss main constructions of the proof. It should be said that technical details of the proofs are systematically omitted, especially when they are not essentially new.

\section{THE GAUSS AUTOMORPHISM: A GEOMETRIC REPRESENTATION}

As is well-known, Gauss automorphism $(\mathbb{Q}, \gamma, \mu)$ (see (1.1)-(1.3)) is an Anosov map isomorphic to a Bernoulli shift with a finite entropy. A natural geometric representation of the Gauss automorphism is given in proposition 2.1 and obtained on the basis of the following construction. Associated with a point $\left(x_{1}, x_{2}\right) \in \mathbb{Q}$ is a broken line $\mathcal{V}$ defined as the boundary $\partial \mathcal{C}$ of the convex hull $\mathcal{C}$ of the set of two-dimensional integer vectors inside a straight angle $\Theta\left(=\Theta\left(x_{1}, x_{2}\right)\right)$, on the half-plane $\left\{\left(X_{1}, X_{2}\right): X_{1}>0\right\}$ ( $X_{1}$ and $X_{2}$ are the plane co-ordinates). Angle $\Theta$ is formed by the half-lines $\mathbb{L}_{+}=$

$\left(\left(X_{1}, X_{2}\right): X_{1}>0, X_{2} / X_{1}=1 / x\right)$ and $\mathbb{L}_{-}=\left(\left(X_{1}, X_{2}\right): X_{1}>0, X_{2} / X_{1}=-x\right)$. Here $x_{1} \sim\left(a_{0}, a_{1}, \ldots\right), x_{2} \sim\left(a_{-1}, a_{-2}, \ldots\right)$, and $x \sim\left(a_{0}, a_{-1}, a_{1}, a_{-2}, \ldots\right)$. More precisely, $\mathcal{V}$ (which, bearing in mind a high-dimensional generalisation, is called a veil), is formed by two broken 'half-lines', $\mathcal{V}_{ \pm}$, with the breaking points in $\mathbb{Z}^{2}$, which approach one of the above straight lines (say $\mathcal{V}_{+}$lies in the quarter-plane $X_{1}, X_{2}>0$ and approaches $\mathbb{L}_{+}$, and $\mathcal{V}_{-}$lies in the quarter-plane $X_{1}>0, X_{2}<0$ and approaches $\left.\mathbb{L}_{-}\right)$. Furthermore, $a_{i}, i \geq 0$, give the integer lengths of the sequence of the segments forming $\mathcal{V}_{+}$, and $a_{i}$, $i \leq-1$, the integer lengths of the sequence of the segments forming $\mathcal{V}_{-}$. (The integer length of a segment (or a vector) with the footpoints in $\mathbb{Z}^{2}$ is defined as the number of the points from $\mathbb{Z}^{2}$ lying in the interior of the segment plus one.) The convex polygon $\mathcal{C}$ is called a Klein polygon.

A standard way to represent the Gauss automorphism is to construct a 'natural' suspension flow whose base automorphism coincides with $(\mathbb{Q}, \gamma, \mu)$. This question was discussed in detail in [AF 1-3] (see also [S]). Our construction seems simpler that in these papers and is carried as follows. The above construction of the veil $\mathcal{V}$ as the boundary of the convex hull of a part of $\mathbb{Z}^{2}$ can be repeated for any cone formed by a pair of lines on $\mathbb{R}^{2}$ through the origin. That is, the veil is geometrically determined by a unit frame $\mathbf{e}$ $=\left(e_{1}, e_{2}\right)$. Here, $e_{1}, e_{2}$ are 2 -vectors with det $\left(e_{1}, e_{2}\right)=1$. An important remark is that the integer lengths of the segments constituting the veil do not change when this frame (or pair of lines) is transformed by an element from the discrete group $\Gamma=\operatorname{SL}(2, \mathbb{Z})$, i.e., by a $2 \times 2$ integer unimodular matrix. As the unit frames are associated with the group SL $(2, \mathbb{R})$ of the $2 \times 2$ real unimodular matrices, we conclude that the phase space $\mathcal{E}$ of the flow is the three-dimensional manifold $G / \Gamma$ where group $G=\operatorname{SL}(2, \mathbb{R})$. That is, an element of $\mathcal{E}$ is an equivalence class, $\mathbf{Y}$, composed by the unit frames $\mathbf{e}^{\prime}$ of the form $\mathbf{e} z$, where $z \in \Gamma$ and $\mathbf{e}$ is a fixed unit frame representing $\mathbf{Y}$. It is convenient to write $\mathbf{e}(\mathbf{Y})$ and $e_{j}(\mathbf{Y}), j=1,2$. By $\mathcal{V}(\mathbf{Y})$ we denote the veil associated with coset $\mathbf{Y}$.

The flow transformations are defined via the left action of the subgroup $\mathrm{D}^{+}(2, \mathbb{R})$ $\subset G$ consisting of the diagonal $2 \times 2$ unimodular matrices with non-negative entries, 
i.e. matrices of the form $\Lambda(\lambda)=\left(\begin{array}{cc}\lambda & 0 \\ 0 & \lambda^{-1}\end{array}\right), \lambda>0$. Namely, if $g \in \mathrm{D}^{+}(2, \mathbb{R})$ then the corresponding transformation $\sigma_{g}: \mathcal{E} \rightarrow \mathcal{E}$ is given by $\sigma_{g} \mathbf{Y}=g \mathbf{Y}$. The invariant measure $\nu$ is the Haar measure of $G$ restricted to $\mathcal{E}$. As $\nu(\mathcal{E})<\infty$ (although $\mathcal{E}$ is not compact), we can assume that $\nu$ is a probability distribution (i.e., $\nu(\mathcal{E})=1$ ).

The dimension of the manifold $\mathrm{D}^{+}(2, \mathbb{R})$ equals one; in other words, $\left\{\sigma_{g}\right\}$ is a oneparameter family of maps $\mathcal{E} \rightarrow \mathcal{E}$. It is convenient to fix an isomorphism $\iota: \mathrm{D}^{+}(2, \mathbb{R}) \rightarrow$ $\mathbb{R}$ by passing to the logarithmic co-ordinates. Here,

$$
\iota \Lambda(\lambda)=\ln \lambda .
$$

With such an isomorphism, we can talk about the one-parameter group $T$ of transformations $\tau_{t}=\sigma_{\iota^{-1} t}, t \in \mathbb{R}$, of $\mathcal{E}$, with the invariant measure $\nu$. The function $\Phi$ : $\mathbf{Y} \rightarrow \mathbb{R}_{+}$establishing the suspension flow structure of $\left\{\sigma_{g}, g \in \mathrm{D}^{+}(2, \mathbb{R})\right\}$ may be defined by

$$
\begin{gathered}
\Phi(\mathbf{Y})=\inf \left[t \geq 0: \text { the vector } e_{1}(\mathbf{Y}) \exp t-e_{2}(\mathbf{Y}) \exp (-t)\right. \text { is } \\
\text { parallel to a segment of the veil determined by frame } \mathbf{e}(\mathbf{Y})] \text {. }
\end{gathered}
$$

Note that vector $e_{1}(\mathbf{Y})(\exp t)-e_{2}(\mathbf{Y})(\exp (-t))$ is parallel to the line through the endpoints of the frame $\Lambda\left(e^{t}\right) \mathbf{e}(\mathbf{Y})$.

Thus, define: (i) $\mathcal{M} \subset \mathcal{E}$ as the zero set of function $\Phi$, (ii) the value $\varphi(\mathbf{Y}), \mathbf{Y} \in \mathcal{M}$, by

$$
\varphi(\mathbf{Y})=\min \left[t: t>0, \tau_{t} \mathbf{Y} \in \mathcal{M}\right]
$$

and (iii) the map $\tau^{0}: \mathcal{M} \rightarrow \mathcal{M}$ by

$$
\mathbf{Y} \mapsto \tau_{\varphi(\mathbf{Y})}(\mathbf{Y}) .
$$

$\mathcal{M}$ is a countable union of manifolds of dimension $\leq 2$. Also, define the probability measure $\nu^{0}$ on $\mathcal{M}$ by using the fact that $\mathrm{D}^{+}(2, \mathbb{R})$ acts in a locally free fashion in a neighbourhood of $\mathcal{M}$. Formally, we (a) write $\nu(d \mathbf{Y})$, where $\mathbf{Y} \in \mathcal{E}$ is of the form $\tau_{t} \mathbf{Y}^{0}$, with $\mathbf{Y}^{0} \in \mathcal{M}$ and $t \in \mathbb{R}$ close to 0 , as $d \widetilde{\nu}^{0}(d \mathbf{Y}) \times d t$, obtaining a measure $\widetilde{\nu}^{0}$ on $\mathcal{M}$, and then (b) normalise $\widetilde{\nu}^{0}$ to get a probability distribution $\nu^{0}$. From the construction it follows that $\nu^{0}$ is invariant under $\tau^{0}$.

Pictorially, a point $\mathbf{Y} \in \mathcal{M}$ is associated with a veil where the segments are labelled by the integers, and the label 0 is attached to the segment parallel to vector $e_{1}(\exp t)-$ $e_{2}(\exp (-t))$. We call it a ground segment and denote it by $\mathbb{P}(\mathbf{Y})$. The map $\tau^{0}$ is represented as the shift of all labels by one. The result of the above construction is the following

Proposition 2.1. The flow $(\mathcal{E}, T, \nu)$ is a suspension flow, with the base automorphism $\left(\mathcal{M}, \tau^{0}, \nu^{0}\right)$. Furthermore, the latter automorphism is isomorphic to the Gauss automorphism $(\mathbb{Q}, \gamma, \mu)$. 
The logarithmic co-ordinates allow us to define the logarithmic projection $\Pi$ of the veil $\mathcal{V}(\mathbf{Y}), \mathbf{Y} \in \mathcal{E}$, to the time-axis $\mathbb{R}^{1}$. Geometrically, we (i) perform the central projection $\Pi_{\text {ce }}$, about the origin, of the segments of $\mathcal{V}(\mathbf{Y})$ (with marked lattice points on them) to the hyperbola $\mathbb{U}(\mathbf{Y})$ given by the equation $X_{1, \mathbf{Y}} X_{2, \mathbf{Y}}=1, X_{1, \mathbf{Y}}, X_{2, \mathbf{Y}}>$ 0 , in the co-ordinates $X_{i, \mathbf{Y}}, i=1,2$, related to frame $\mathbf{e}(\mathbf{Y})$, and then (ii) pass to the coordinate $t=\ln x_{1, \mathbf{Y}}$ on $\mathbb{U}(\mathbf{Y})$. As a result, we obtain a partition of $\mathbb{R}^{1}$ into disjoint intervals (the endpoints of these intervals are the images, under projection $\Pi_{\mathrm{ce}}$, of the vertices of $\mathcal{V}(\mathbf{Y})$, with marked points on them (which are the images of the lattice points lying inside the segments of $\mathcal{V}(\mathbf{Y})$ ). The intervals constituting the partition are called cells (again in view of future multi-dimensional generalisations). The image of a vertex of $\mathcal{V}(\mathbf{Y})$ is also called a vertex (of a cell partition).

It is convenient to label the cells forming a partition by the integers. If $\mathbf{Y} \in \mathcal{M}$, there exists a distinguished cell which is the projection, under $\Pi$, of the ground segment $\mathbb{P}(\mathbf{Y})$ of $\mathcal{V}$; such a cell is labelled by 0 . Otherwise, i.e., when $\mathbf{Y} \notin \mathcal{M}$, there is a distinguished pair of adjacent cells (or, if one prefers, a vertex of the partition). This vertex (also called ground) is the image, under $\Pi$, of the vertex of $\mathcal{V}(\mathbf{Y})$ which is the first one to be reached by the translations, towards the veil, of the line $\mathbb{G}(\mathbf{Y})$ given by the equation $\left\langle x, e_{1}(\mathbf{Y})\right\rangle+\left\langle x, e_{2}(\mathbf{Y})\right\rangle=0$. Note that $\mathbb{G}(\mathbf{Y})$ is a line through the origin, parallel to vector $e_{1}(\mathbf{Y})-e_{2}(\mathbf{Y})$. One of two cells adjacent to the ground vertex, say the furthest to the right (in the sense of the direction of co-ordinate $t$ ) is labelled by 0 .

Once the 0 -th label is attached, we assign the numbers $j \in \mathbb{Z}^{1}$ observing the following principle: two adjacent cells receive labels that differ by one, and the furthest right cell of the adjacent pair receives the greater label.

As a result, a point $\mathbf{Y} \in \mathcal{M}$ is identified with a sequence $\mathbf{a}=\left(a_{i}, i \in \mathbb{Z}^{1}\right)$ where $a_{i}$ is the number of the marked points inside the $i$ th cell plus one. We also can define a function $\phi$, on $\mathcal{M}$, whose value $\phi(\mathbf{a})>0$ is determined by the condition that vector $e_{1}(\mathbf{Y}) \exp (\phi(\mathbf{a}))+e_{2}(\mathbf{Y}) \exp (-\phi(\mathbf{a}))$ is parallel to the next-to-the-ground segment of the veil, namely the one projected to cell one. On the other hand, a point $\mathbf{Y} \in \mathcal{E} \backslash \mathcal{M}$ is identified with a pair $\left(\mathbf{a}, t^{0}\right)$ where $\mathbf{a}$ is as above, and $0<t^{0}<\phi(\mathbf{a})$ is determined by the condition that vector $e_{1}(\mathbf{Y})\left(\exp t^{0}\right)+e_{2}(\mathbf{Y})\left(\exp \left(-t^{0}\right)\right)$ is again parallel to the segment of the veil which is projected to cell one.

Proposition 2.2. Given above identification, flow $(\mathcal{E}, T, \nu)$ is isomorphic to the suspension flow with the base automorphism $(\mathbb{Q}, \gamma, \mu)$ and the function $\phi$.

The proof of Proposition 2.2 follows the arguments developed in [AF 1-3] and is omitted.

A straightforward calculation with probability distribution (1.3), (1.4) leads to the following asymptotical formulas. Let $N_{s}(\mathbf{Y}, R), s \in \mathbb{Z}_{+}$, denote the number of edges of veil $\mathbf{Y}$ containing $s+1$ lattice points and lying within a circle in $\mathbb{R}^{2}$ of radius $R>0$ centered at the origin, and $N(\mathbf{Y}, R)$ the total number of edges within this circle. Then 
for $\nu$-a.s. $\mathbf{Y} \in \mathcal{E}$,

$$
N_{s}(\mathbf{Y}, R) \simeq \frac{2 \ln R}{\zeta(2)} \ln \frac{(s+1)^{2}}{s(s+2)}
$$

and

$$
N(\mathbf{Y}, R) \simeq \ln 2 \frac{2 \ln R}{\zeta(2)}
$$

Our approach may be extended to the case of 'larger' groups, where $G=\operatorname{SL}(2, \mathbb{R}) \ltimes$ $\mathbb{R}^{2}$ (the group of the affine transformations of $\mathbb{R}^{2}$ ) and $\Gamma=\operatorname{SL}(2, \mathbb{Z}) \ltimes \mathbb{Z}^{2}$, with the same group $T$ as before. Pictorially, it means that the corner point of an angle is not necessarily at zero. Similar calculations show that if the corner is distributed with a density on unit square $\mathbb{Q}$ then

$$
N_{s}(\mathbf{Y}, R) \simeq \frac{\ln R}{\zeta(2)} \ln \frac{(s-1)^{s-1}(s+1)^{3(s+1)}}{s^{3 s}(s+2)^{s+2}},
$$

whereas $N(\mathbf{Y}, R)$ has the same asymptotic (2.5). If the corner is fixed at a rational point, the limit of $N_{s}(\mathbf{Y}, R) / \ln R$ may be calculated explicitly. The resulting formula is quite cumbersome, but the total number $N_{s}(\mathbf{Y}, R)$ again behaves as in $(2.5)$. We conjecture that $N_{s}(\mathbf{Y}, R)$ has the same asymptotic as before for any position of the corner on $\mathbb{R}^{2}$.

\section{SIMPLICIAL VEILS IN HIGHER DIMENSIONS}

We begin with a dynamical system that is a natural generalisation of flow $(\mathcal{E}, T, \nu)$ (it is convenient to use the system of notation from the preceding section). A veil in the $n$-dimensional space $\mathbb{R}^{n}$ is constructed in a similar fashion. Given a simplicial cone, we take the convex hull $\mathcal{C}$ of the set of integer points lying inside the cone; the veil $\mathcal{V}$ is the boundary of $\mathcal{C}$. It is an $(n-1)$-dimensional polyhedral surface 'approaching' the boundary of the simplicial cone. The plane pieces of this surface are called faces; each face is in turn a convex polyhedron; its boundary is decomposed into plain faces of dimension $n-2$, etc. As before, we consider veils modulo the action of the group $\Gamma=$ $\mathrm{SL}(n, \mathbb{Z})$. For simplicity, the corresponding equivalence classes are again called veils; we also use for such a class the previous notation $\mathcal{V}$. The simplicial cone is determined by the unit frame $\mathbf{e}=\left(e_{1}, \ldots, e_{n}\right)$ (with $\operatorname{det}\left(e_{1}, \ldots, e_{n}\right)=1$ ); thus the phase space of our dynamical system is the $\left(n^{2}-1\right)$-dimensional fundamental domain $\mathcal{E}=G / \Gamma$ where $G=$ $\mathrm{SL}(n, \mathbb{R})$. A point of $\mathcal{E}$ is denoted by $\mathbf{Y}$, and is a coset $\mathbf{e} z, z \in \Gamma$, where $\mathbf{e}=\left(e_{1}, \ldots, e_{n}\right)$ is a unit frame representing $\mathbf{e}$. As before, we use the notation $\mathbf{Y} \sim(\mathbf{e}), \mathbf{e}(\mathbf{Y})$ and $e_{j}(\mathbf{Y})$, $j=1, \ldots, n$. Furthermore, we again denote by $\mathcal{V}(\mathbf{Y})$ the veil associated with coset $\mathbf{Y}$.

The flow transformations on $\mathcal{E}$ are defined, as before, via the left action of the group $\mathrm{D}^{+}(n, \mathbb{R})$ of the real $n \times n$ unimodular diagonal matrices with non-negative entries i.e. the matrices of the form

$$
\Lambda(\underline{\lambda})=\left(\begin{array}{ccccc}
\lambda_{1} & 0 & 0 & \ldots & 0 \\
0 & \lambda_{2} & 0 & \ldots & 0 \\
& & & \ldots & \\
0 & 0 & 0 & \ldots & \lambda_{n}
\end{array}\right), \underline{\lambda}=\left(\lambda_{1}, \ldots, \lambda_{n}\right), \lambda_{1}, \ldots, \lambda_{n}>0, \lambda_{1} \ldots \lambda_{n}=1,
$$


namely, $\sigma_{\Lambda(\underline{\lambda})}: \mathbf{Y} \in \mathcal{E} \mapsto \Lambda(\underline{\lambda}) \mathbf{Y}$. The invariant measure $\nu$ is again the Haar measure of $G$ restricted to $\mathcal{E}$. As before, $\nu(\mathcal{E})<\infty$ (see [B]); we again assume that $\nu$ is a probability distribution.

The dimension of the manifold $\mathrm{D}^{+}(n, \mathbb{R})$ equals $n-1$. The logarithmic co-ordinates on $\mathrm{D}^{+}(n, \mathbb{R})$ may be introduced via an isomorphism $\iota: \mathrm{D}^{+}(2, \mathbb{R}) \rightarrow \mathbb{R}^{n-1}$ where

$$
\iota \Lambda(\underline{\lambda})=\left(\ln \lambda_{1}, \ldots, \ln \lambda_{n-1}\right) .
$$

With such an isomorphism, we can talk about the group of transformations $\tau_{\underline{t}}=\sigma_{\iota^{-1}}$, $\underline{t}=\left(t_{1}, \ldots, t_{n-1}\right) \in \mathbb{R}^{n-1}$, on $\mathcal{E}$, with the invariant measure $\nu$. It is well-known (see [M $1]$, [Z]) that flow $(\mathcal{E}, T, \nu)$ is ergodic. However, it is not difficult to check that its $(n-1)$ dimensional entropy equals zero. The same is true of the $k$-dimensional entropy (i.e., the entropy of the action of a $k$-parameter subgroup $\tau_{\underline{t}}, \underline{t} \in \mathbb{H}^{k}$, where $\mathbb{H}^{k}$ is a $k$-dimensional subspace in $\left.\mathbb{R}^{n-1}, 1<k<n-1\right)$. On the other hand, the one-dimensional entropy (i.e., the entropy of the action of a one-parameter subgroup $\gamma_{\underline{t}}, \underline{t} \in \mathbb{H}^{1}$, where $\mathbb{H}^{1}$ is a straight line in $\mathbb{R}^{n-1}$ ) is positive.

Flow $(\mathcal{E}, T, \nu)$ admits a kind of a suspension flow representation. Namely, let $\mathbb{G}(\mathbf{Y})$ be the $(n-1)$-dimensional hyperplane consisting of vectors of the form $\sum \mu_{i} e_{i}(\mathbf{Y})$ where $\sum \mu_{i}=0$. Denote by $\mathcal{M}$ the following subset of $\mathcal{E}$ :

$$
\mathcal{M}=\{Y \in \mathcal{E}: \quad \text { one of the }(n-1)-\text { dimensional faces }
$$

of the veil $\mathcal{V}(\mathbf{Y})$ is parallel to the hyperplane $\mathbb{G}(\mathbf{Y})\}$.

Let $\mathbb{P}(\mathbf{Y}), \mathbf{Y} \in \mathcal{M}$, denote the convex $(n-1)$-dimensional polyhedron that is the part of veil $\mathcal{V}(\mathbf{Y})$ lying on the face figuring in the definition of $\mathcal{M}$ (more precisely, the whole class of the polyhedra obtained by the action of $\operatorname{SL}(n-1, \mathbb{Z}) \ltimes \mathbb{Z}^{n-1}$, the group of the affine integer transforms of $\left.\mathbb{R}^{n-1}\right) . \mathbb{P}(\mathbf{Y})$ is called the ground polyhedron, or ground face (of veil $\mathcal{V}(\mathbf{Y}), \mathbf{Y} \in \mathcal{M}$ ). $\mathcal{M}$ is a countable union of submanifolds of $\mathcal{E}$ of dimension $n(n-1)$. The probability measure $\nu^{0}$ on $\mathcal{M}$ is again constructed from measure $\nu$ by using the fact that $\mathcal{M}$ is transversal to the orbits $\left\{\gamma_{\underline{t}}, \underline{t} \in \mathbb{R}^{n-1}\right\}$.

However, instead of a single basic map $\gamma^{0}: \mathcal{M} \rightarrow \mathcal{M}$ we now have, for $\nu^{0}$-almost all $\mathbf{Y} \in \mathcal{M}$, a collection of maps

$$
\gamma_{1, \mathbf{Y}}^{0}, \ldots, \gamma_{b(\mathbf{Y}), \mathbf{Y}}^{0}: \mathcal{M} \rightarrow \mathcal{M}
$$

preserving measure $\nu^{0}$. Here, the numbers $1, \ldots, b(\mathbf{Y})$ label the $(n-2)$-dimensional faces forming the boundary $\partial \mathbb{P}$. Pictorially speaking, each map $\gamma_{j, \mathbf{Y}}^{0}, j=1, \ldots, b(\mathbf{Y})$, takes the veil $\mathcal{V}(\mathbf{Y})$ to a veil where the ground polyhedron is the one adjacent to $\mathbb{P}(\mathbf{Y})$ via the $j$ th face of $\partial \mathbb{P}(\mathbf{Y})$, and the 'combinatorial structure' of $\mathcal{V}(\mathbf{Y})$ is preserved.

The combinatorial structure of the veil may be described, as before, in terms of a logarithmic projection $\Pi$. Namely, we again take (i) the central projection $\Pi_{\text {ce }}$, about 
the origin, of the $(n-1)$-dimensional faces of $\mathcal{V}(\mathbf{Y})$ (with marked lattice points on them) to the hyperboloid $\mathbb{U}(\mathbf{Y})$ given by the equation $X_{1, \mathbf{Y}} \ldots X_{n, \mathbf{Y}}=1, X_{1, \mathbf{Y}}, \ldots, X_{n, \mathbf{Y}}>0$, in the co-ordinates $X_{i, \mathbf{Y}}, i=1, \ldots, n$, related to frame $\mathbf{e}(\mathbf{Y})$, and then (ii) pass to the coordinates $\underline{t}=\left(\ln X_{1, \mathbf{Y}}, \ldots, \ln X_{n-1, \mathbf{Y}}\right)$ on $\mathbb{U}(\mathbf{Y})$. As in Section 2, we obtain a partition of the linear time-manifold $\mathbb{R}^{n-1}$ into disjoint domains, with marked points on them (which are the images of the lattice points lying on the faces of $\mathcal{V}(\mathbf{Y})$ ). The boundaries of these domains are the images, under $\Pi$, of the faces of $\mathcal{V}(\mathbf{Y})$ of lower dimension (down to the vertices). The domains constituting the partition (together with the collections of lattice points in them) are called cells, and the images of the vertices of $\mathcal{V}(\mathbf{Y})$ are again called vertices.

Unlike the two-dimensional case, for $n \geq 3$ there is in general no lattice-like labelling of the cells of the given partition. However, for a point $\mathbf{Y} \in \mathcal{M}$ we can pick a particular cell, and for a generic point $\mathbf{Y} \in \mathcal{E} \backslash \mathcal{M}$ a particular vertex. Namely, if $\mathbf{Y} \in \mathcal{M}$ then the distinguished cell is the image, under $\Pi$, of the ground polyhedron $\mathbb{P}(\mathbf{Y})$ (we call it a ground cell). On the other hand, if $\mathbf{Y} \notin \mathcal{M}$, then the distinguished vertex (also called ground) is the one we first meet by translating the $(n-1)$-dimensional hyperplane $\mathbb{G}(\mathbf{Y})$ in the direction of $\mathcal{V}(\mathbf{Y})$. Natural parameters here are the area of the ground cell for $\mathbf{Y} \in \mathcal{M}$ and the total area of the cells incident to the ground vertex for a generic $\mathbf{Y} \in \mathcal{E} \backslash \mathcal{M}$.

One of the first questions about flow $(\mathcal{E}, T, \nu)$ which one can try to address is about the 'statistical' properties of ground polyhedron $\mathbb{P}(\mathbf{Y}), \mathbf{Y} \in \mathcal{M}$, with respect to measure $\nu^{0}$. Denote by $\mathcal{M}_{s}$ the subset of $\mathcal{M}$ where $\mathbb{P}(\mathbf{Y})$ contains $s$ lattice points, and set $\rho_{s}^{0}=\nu^{0}\left(\mathcal{M}_{s}\right), s \in \mathbb{Z}_{+}$. In this paper we establish the following assertion.

Theorem 1. The expected value

$$
E^{0}=\sum_{s \in \mathbb{Z}_{+}} s \rho_{s}^{0}=\infty .
$$

Pictorially, the assertion of Theorem 1 may be interpreted as the statement that 'on average' the number of the lattice points on a face (or in a cell), of a $\nu^{0}$-'typical' veil $\mathcal{V}(\mathbf{Y}), \mathbf{Y} \in \mathcal{M}$, is infinite. The precise form of such a statement may vary with the choice of the concept of averaging. We comment here on two (from our point of view, most natural) concepts: the sample average and the log-volume-weighted average. Here, one takes a 'large' domain, in $\mathbb{R}^{n-1}$ (say a ball of a big radius $R$ centred at the origin), and counts the numbers of the integer points within the cells of the veil which are entirely inside the domain. In the case of the sample average the total number of the integer points is divided by the number of cells. In the case of the log-volume-weighted average it is divided by the volume of the domain. One then passes to the limit as the domain is inflated to cover eventually all $\mathbb{R}^{n-1}$.

Theorem 2. Set $N_{R}^{1}(\mathbf{Y})=\frac{M_{R}(\mathbf{Y})}{m_{R}(\mathbf{Y})}$ and $N_{R}^{2}(\mathbf{Y})=\frac{M_{R}(\mathbf{Y})}{v_{R}}$, where $M_{R}(\mathbf{Y})$ is the total number of lattice points in cells that are entirely inside ball $\mathbb{B}_{R} \subset \mathbb{R}^{n-1}$ of radius $R$ 
centred at the origin, and $m_{R}(\mathbf{Y})$ is the number of such cells, and $v_{R}$ is the (Euclidean) volume of $\mathbb{B}_{R}$. Then, with $\nu$-probability one,

$$
E^{1}:=\lim _{R \rightarrow \infty} N_{R}^{1}(\mathbf{Y})=\infty, \text { and } E^{2}:=\lim _{R \rightarrow \infty} N_{R}^{2}(\mathbf{Y})=\infty
$$

For the proof of Theorems 1 and 2, see Section 5. Formulas (3.5) and (3.6) extend relation (1.3) to the multidimensional case. It is a challenging problem to determine the distribution of the random variable $N$ on $\mathcal{M}$ (cf. (1.2)): at the moment this seems beyond our reach. Numerical results approximating this distribution will be published separately.

A similar problem arises with other parameters of the (random) polygon $\mathbb{P}(\mathbf{Y})$ : the total number of lattice points on the boundary $\partial \mathbb{P}(\mathbf{Y})$, the number of lattice points lying on the faces of $\partial \mathbb{P}(\mathbf{Y})$ of dimension $n-3$, etc., and finally the number of vertices in $\mathbb{P}(\mathbf{Y})$. Similarly, one can consider the distribution of the number of vertices of $\mathbb{P}(\mathbf{Y})$ of a given degree $r$ (including or excluding the edges of faces adjacent to $\mathbb{P}(\mathbf{Y})$ ), the number of edges with vertices of given degrees $\left(r, r^{\prime}\right)$, etc. In general, one may speak of the distribution of any 'local' combinatorial structure of veil $\mathcal{V}(\mathbf{Y})$. In this paper, we will comment on the distribution of the number of vertices of $\mathbb{P}(\mathbf{Y})$. The following assertion is proved in Section 5 .

Lemma 3.1. There exists a constant, $\delta(=\delta(d))>0$, such that for any two distinct vertices $v, v^{\prime}$ of veil $\mathcal{V}(\mathbf{Y})$ the log-distance $\operatorname{dist}_{\mathbb{R}^{n-1}}\left(\Pi v, \Pi v^{\prime}\right):=\left\|\Pi v-\Pi v^{\prime}\right\| \geq \delta$.

Lemma 3.1, combined with a thorough case-by-case analysis of a number of geometric situations, leads to

Theorem 3. Let $V(=V(\mathbf{Y}))$ denote the number of vertices in $\mathbb{P}(\mathbf{Y}), \mathbf{Y} \in \mathcal{M}$. Then, for positive constants $c$ and $c^{ \pm}$,

$$
c^{-} \leq \nu^{0}(V(\mathbf{Y})=s) \exp \left(c s^{1 /(n-2)}\right) \leq c^{+}
$$

We conclude this section with a brief discussion of possible algorithms, for finding a rational (or integer) approximations, which can be designed on the basis of the approach proposed in this paper. Let $\mathbb{K} \subset \mathbb{R}^{n}$ be a linear subspace, of dimension $l<n$; we consider first the problem of finding a point $m \in \mathbb{Z}^{n}$ close to $\mathbb{K}$. Fix a 'nice' set, e.g. a $d$-dimensional ball $\mathbb{B}$ centred at the origin and of a sufficiently large radius. The basic idea is simple: given $\epsilon>0$, we apply a linear transformation $A_{\epsilon, \mathbb{K}} \in G$ to $\mathbb{B}$ and get a 'tube-like' domain (e.g. an ellipsoid) $\mathbb{T}=A_{\epsilon, \mathbb{K}} \mathbb{B} \subset \mathbb{R}^{n}$ 'stretched' along $\mathbb{K}$ so that the width of $\mathbb{T}$ in the directions orthogonal to $\mathbb{K}$ is $<\epsilon$. The transformation $A_{\epsilon, \mathbb{K}}$ can be specified to have $\mathbb{K}$ as the eigenspace with the eigenvalue of size $\sim \epsilon^{-\frac{n-l}{l}}$ and the orthogonal complement $\mathbb{K}^{\perp}$ as the eigenspace with the eigenvalue of size $\sim \epsilon$. If the volume of $\mathbb{B}$ (which is equal to the volume of $\mathbb{T}$ ) is sufficiently large, then by Minkowski's convex body theorem (see, e.g., $[\mathrm{C}]$ and $[\mathrm{GL}]$ ), $\mathbb{T}$ will contain a non-zero integer point. 
This is be a point which we look for. One can also formulate the question other way around: find a point in the unimodular lattice $A_{\epsilon, \mathbb{K}}^{-1}\left(\mathbb{Z}^{n}\right)$ inside the body $\mathbb{B}$.

Observe that transformations $A_{\epsilon, \mathbb{K}}$ form a one-parameter subgroup of $G$. Thus, our problem is reduced to that of calculating the action of a 'large' element of such a subgroup at a point of $\mathcal{E}$. This can be implemented numerically by using a partition of $\mathcal{E}$ into disjoint semi-algebraic sets.

If we want to find a rational approximation for a given vector $\theta=\left(\theta_{1}, \ldots, \theta_{n}\right)$, we form a $(n+1)$-dimensional vector $\bar{\theta}=\left(\theta_{1}, \ldots, \theta_{n}, 1\right)$ and repeat the above construction for the one-dimensional space $\mathbb{K} \subset \mathbb{R}^{n+1}$ along $\bar{\theta}$. Then an integer $\epsilon / 2$-approximation for $\mathbb{K}$ will straightforwardly lead to an $\epsilon$ - approximation for $\theta$. It is not hard to assess the accuracy of such an algorithm: its speed will be similar to that of the algorithm proposed in $[\mathrm{La}]$.

\section{PARABOLIC VEILS}

We now take $G=\mathrm{SL}(n, \mathbb{R}) \ltimes \mathbb{R}^{n}, \Gamma=\mathrm{SL}(n, \mathbb{R}) \ltimes \mathbb{Z}^{n}$. Group $T$ will be of the form $\mathrm{SO}(n-1, \mathbb{R}) \ltimes \mathbb{R}^{n-1}$ and identified with a subgroup of $G$ generated by the following transformations $\pi .: \mathbb{R}^{n} \rightarrow \mathbb{R}:$

$$
\begin{gathered}
\pi_{\underline{t}}:\left(x_{1}, \ldots, x_{n-1}, x_{n}\right) \mapsto\left(x_{1}+t_{1}, \ldots x_{n-1}+t_{n-1}, x_{n}+\sum_{1 \leq i \leq n-1}\left(2 t_{i} x_{i}+t_{i}^{2}\right)\right), \\
\underline{t}=\left(t_{1}, \ldots t_{n-1}\right) \in \mathbb{R}^{n-1}, \\
\pi_{A}:\left(x_{1}, \ldots, x_{n-1}, x_{n}\right) \mapsto\left(\sum_{1 \leq j \leq n-1} a_{1, j} x_{j}, \ldots, \sum_{\substack{1 \leq j \leq n-1 \\
A}} a_{n-1, j} x_{j}, x_{n}\right) \\
A=\left(a_{i j}\right) \in \operatorname{SO}(n-1, \mathbb{R}) .
\end{gathered}
$$

These transformations preserve the paraboloid $\left\{x_{n}=\sum_{1 \leq j \leq n-1} x_{j}^{2}\right\}$. As before, $\mathcal{E}=$ $G / \Gamma$. Pictorially, a point $\mathbf{Y} \in \mathcal{E}$ is associated with a lattice of the unit volume in $\mathbb{R}^{n}$, not necessarily containing the origin.

As before, let $\nu$ be the probability measure on $\mathcal{E}$ generated by the Haar measure on $G$. We are interested in the flow $(\mathcal{E}, T, \nu)$ (with the left action of group $T$ ). As follows from Theorem 4 below, this flow is ergodic (see also [M 2] where a more general assertion is established). However, for $n \geq 2$ its restriction to any 1-parameter subgroup $\mathbb{R} \subset \mathbb{R}^{n-1} \subset T$ is not Bernoulli (it has zero entropy).

Theorem 4. Any non-compact closed subgroup $T^{\prime} \subseteq T$ acts ergodically on $(G / \Gamma, \nu)$ (including $T$ itself).

As before, we consider the lattice points in the domain $X_{n} \geq \sum_{1 \leq j \leq n-1} X_{j}^{2}$ (i.e., inside the paraboloid) and denote by $\mathcal{C}(\mathbf{Y})$ the convex hull of these; the boundary $\partial \mathcal{C}(\mathbf{Y})$ 
is again called a (parabolic) veil and denoted $\mathcal{V}(\mathbf{Y})$. Set

$$
\mathcal{M}=\left\{Y \in \mathcal{E}: \text { veil } \mathcal{V}(\mathbf{Y}) \text { contains a horizontal face, where } x_{n} \text { is constant }\right\}
$$

Such a face is denoted $\mathbb{P}(\mathbf{Y})$ and again called the ground polyhedron, or ground face (of veil $\mathcal{V}(\mathbf{Y}), \mathbf{Y} \in \mathcal{M})$. Furthermore, it is convenient to consider a subset $\mathcal{M}_{s} \subset \mathcal{M}$ where the ground face contains $s$ lattice points.

In the same fashion as before we introduce a probability measure $\nu^{0}$ on $\mathcal{M}$. The probabilities

$$
\rho_{s}^{0}=\nu^{0}\left(\mathcal{M}_{s}\right), \quad s=1,2, \ldots,
$$

may be considered as a 'parabolic' analogue of 'simplicial' probabilities (1.4) and the expected value

$$
E^{0}=\sum_{s \in \mathbb{Z}_{+}} s \rho_{s}^{0}
$$

as the analogue of (1.5). However, in contrast to the simplicial case, the expected values are finite.

Theorem 5. For parabolic veils, in any dimension $n \geq 2$,

$$
E^{0}<\infty
$$

The proof of Theorem 5 is based on a version of Minkowski's convex body theorem: apart from some 'degenerate' cases, the number of lattice points on a face is $\leq c_{0}$ times the $(n-1)$-dimensional volume of the face, where $c_{0}\left(=c_{0}(n)\right)$ is a constant. By direct calculation, both the expected value of the $(n-1)$-volume of $\mathbb{P}(\mathbf{Y})$ and the contribution from the degenerate cases are finite, which leads to (4.4).

Theorem 6. Consider the projection $\mathbb{R}^{n} \rightarrow \mathbb{R}^{n-1}$ given by $\left(X_{1}, \ldots, X_{n-1}, X_{n}\right)$ $\mapsto\left(X_{1}, \ldots, X_{n-1}\right)$. As in the simplicial case, set $N_{R}^{1}(\mathbf{Y})=\frac{M_{R}(\mathbf{Y})}{m_{R}(\mathbf{Y})}$ and $N_{R}^{2}(\mathbf{Y})=$ $\frac{M_{R}(\mathbf{Y})}{v_{R}}$, where $M_{R}(\mathbf{Y})$ is the total number of the integer points in the cells that are projected entirely inside the ball $\mathbb{B}_{R} \subset \mathbb{R}^{n-1}$ of radius $R$ centred at the origin, $m_{R}(\mathbf{Y})$ is the number of such cells, and $v_{R}$ is the Euclidean volume of $\mathbb{B}_{R}$. Then, for parabolic veils, in any dimension $n \geq 2$, with $\nu$-probability one, the limits

$$
E^{1}=\lim _{R \rightarrow \infty} N_{R}^{1}(\mathbf{Y}) \text { and } E^{2}=\lim _{R \rightarrow \infty} N_{R}^{2}(\mathbf{Y})
$$

are finite constants. The ratio $r=r(n):=E^{2} / E^{1}$ is the average number of cells per unit volume in the othogonal projection of the veil to the horizontal hyperplane $\mathbb{R}^{n-1}$.

The proof of Theorem 6 combines arguments from that of Theorem 5 , Theorem 4 and Theorem 2 (see Section 5). 
In the case $n=2$, flow $(\mathcal{E}, T, \nu)$ is a suspension flow, with the base automorphism $\left(\mathcal{M}, \phi^{0}, \nu^{0}\right)$ where $\phi^{0}$ is the Poincare return flow map $\mathcal{M} \rightarrow \mathcal{M}$. Following the analogy with the simplicial case, we call $\left(\mathcal{M}, \phi^{0}, \nu^{0}\right)$ a parabolic Gauss automorphism. It would be interesting to find a 'natural' realisation of this automorphism on a 'nice' four-dimensional manifold. In this paper we give 'more explicit' formulas for parabolic probabilities $\rho_{s}$ :

Theorem 7. For $n=2$,

$$
\rho_{s}^{0} \simeq c s^{-7 / 3}
$$

where $c$ is a positive constant.

One can calculate numerically appropriate integrals and obtain that, for example, the average density $r(2)$ of cells in Theorem 6 for $n=2$ is $\simeq 0.69254 \ldots$.

There are several alternative approaches to studying statistical properties of the parabolic veil. For example, given $\mathbf{Y} \in \mathcal{E}$, we can specify a face of $\mathcal{V}(\mathbf{Y})$ which is closest to the origin $O=(0, \ldots, 0) \in \mathbb{R}^{n}$; apart from the set of $\nu$-measure zero, this is a welldefined object which we denote by $\mathbb{P}^{\text {ne }}(Y)$. Furthermore, denote by $N^{\text {ne }}(\mathbf{Y})$ the number of the lattice points inside $\mathbb{P}^{n e}(Y)$. Finally, denote by $\Sigma(\mathbf{Y})$ the $(n-1)$-dimensional Euclidean area of the set $\left\{\underline{t}=\left(t_{1}, \ldots, t_{n-1}\right) \in \mathbb{R}^{n-1}: \mathbb{P}^{n e}(Y)=\mathbb{P}^{n e}\left(\pi_{\underline{t}} \mathbf{Y}\right)\right\}$.

Theorem 8. The expected values

$$
E^{\mathrm{ne}}=\int_{\mathcal{E}} \mathrm{d} \nu(\mathbf{Y}) N^{\mathrm{ne}}(\mathbf{Y})
$$

and

$$
S^{\text {ne }}=\int_{\mathcal{E}} d \nu(\mathbf{Y}) \Sigma^{\text {ne }}(\mathbf{Y})
$$

are both finite.

We conclude this section with a theorem emphasising the significance of parabolic veils. First, note that the above construction can be repeated for any paraboloid $x_{n}=$ $\mathbf{Q}\left(x_{1}, \ldots, x_{n-1}\right)$ where $\mathbf{Q}$ is a positive definite quadratic form. The corresponding expected values (4.7), (4.8) will be denoted by $E^{\text {ne }}(\mathbf{Q})$ and $S^{\text {ne }}(\mathbf{Q})$, respectively. Consider a convex domain $\mathbb{D} \subset \mathbb{R}^{n}$, with a $C^{2}$-smooth boundary $\partial \mathbb{D}$, such that the origin $O \in \mathbb{D}$. Denote by $\mathbf{Q}(\mathbf{x})$ the second quadratic form of $\partial \mathbb{D}$ at point $\mathbf{x} \in \partial \mathbb{D}$ and by $v$ the volume element on $\partial \mathbb{D}$ induced by the Riemannian metric of the ambient space $\mathbb{R}^{n}$. We assume that the form $\mathbf{Q}(\mathbf{x})$ is strictly positive at each point $x \in \partial \mathbb{D}$. Given $\lambda>0$, consider a rescaled domain $\lambda \mathbb{D}=\left\{\mathbf{x} \in \mathbb{R}^{n}: \lambda^{-1} \mathbf{x} \in \mathbb{D}\right\}$. Now, given $\mathbf{Y} \in \mathcal{E}$, consider the boundary $\widetilde{\mathcal{V}}(\lambda, \mathbf{Y})$ of the convex hull $\widetilde{\mathcal{C}}(\lambda, \mathbf{Y})$ of the set of lattice points inside $\lambda \mathbb{D}$. Furthermore, given $\mathbf{x} \in \partial \mathbb{D}$, let $N^{\text {ne }}(\mathbf{x}, \lambda, \mathbf{Y})$ denote the number of lattice points lying on the face of $\widetilde{\mathcal{V}}(\lambda, \mathbf{Y})$ which is closest to point $\lambda \mathbf{x} \in \partial(\lambda \mathbb{D})$ (if there are two or more such faces, we sum over them). Finally, $\bar{N}(\lambda, \mathbf{Y})$ will denote the total number of faces of $\widetilde{\mathcal{V}}(\lambda, \mathbf{Y})$. 


\section{Theorem 9.}

(i) For $\nu$-a.a. $\mathbf{Y} \in \mathcal{E}$

$$
\lim _{\lambda \rightarrow \infty} \lambda^{-n(n-1) /(n+1)} \bar{N}(\lambda, \mathbf{Y})=r(n) \int_{\partial \mathbb{D}} \mathrm{d} v(\mathbf{y}) \sqrt{\operatorname{det} \mathbf{Q}(\mathbf{y})}
$$

(ii) There exists a positive constant $c(=c(n))$ such that for any $\mathbf{x} \in \partial \mathbb{D}$, and for $\nu$-a.a. $\mathbf{Y} \in \mathcal{E}$

$$
\lim _{\lambda \rightarrow \infty} \lambda^{-n(n-1) /(n+1)} N(\mathbf{x}, \lambda, \mathbf{Y})=c \sqrt{\operatorname{det} \mathbf{Q}(\mathbf{y})}
$$

The proof of Theorem 9 is based on the ergodicity property stated in Theorem 4 and additional straightforward (although tedious) estimates. The main idea is that after applying affine transformations preserving the volume element, one can approximate large pieces of $\partial(\lambda \mathbb{D})$ by standard paraboloids.

A 'dual' point of view is also possible: given $\lambda>0$ and $\mathbf{Y} \in \mathcal{E}$, pick up points $\mathbf{x} \in \partial \mathbb{D}$ such that the tangent hyperplane $\mathbb{T}(\lambda, \mathbf{x})$ through $\lambda \mathbf{x}$ to $\partial(\lambda \mathbb{D})$ is parallel to a face of $\widetilde{\mathcal{V}}(\lambda, \mathbf{Y})$. This gives a (finite) set $\mathbf{X}=\{\mathbf{x}\} \subset \partial \mathbb{D}$; the face associated with $\mathbf{x}$ is denoted by $\mathbb{F}_{\lambda}(\mathbf{x}, \mathbf{Y})$. We can also consider functions $\xi: \mathbf{x} \in \mathbf{X} \mapsto \xi(\mathbf{x}) \in \mathbb{Z}_{+}$ describing the number of lattice points on $\mathbb{F}_{\lambda}(\mathbf{x}, \mathbf{Y})$, the number of vertices in $\mathbb{F}_{\lambda}(\mathbf{x}, \mathbf{Y})$, etc.; in principle, any 'local' combinatorial characteristic of the veil may be considered. For definiteness, assume that $\xi(\mathbf{x})$ equals the number of lattice points in $\mathbb{F}_{\lambda}(\mathbf{x}, \mathbf{Y})$. As $\mathbf{Y}$ varies along probability space $(\mathcal{E}, \nu)$, this defines a random 'marked' point field $\Xi_{\lambda}$ on $\partial \mathbb{D}$, with marks from $\mathbb{Z}_{+}$; a sample of such a random field is a pair $(\mathbf{X}, \psi)$. The distribution of $\Xi_{\lambda}$ is determined by a sequence of its moment measures $\kappa_{m}, m \in \mathbb{Z}_{+}$. Here $\kappa_{m}$ is a Borel measure on $(\partial \mathbb{D})^{m} \times \mathbb{Z}_{+}^{m}$ defined by

$$
\kappa_{m}(B)=\int_{\mathcal{E}} \nu(\mathrm{d} \mathbf{Y}) N_{B}(\mathbf{Y})
$$

where $B \subseteq \partial \mathbb{D}^{m} \times \mathbb{Z}_{+}^{m}$, and $N_{B}(\mathbf{Y})$ is the number of pairs of 'vectors' $\left(\left(\mathbf{x}_{1}, \ldots, \mathbf{x}_{m}\right)\right.$, $\left.\left(n_{1}, \ldots, n_{m}\right)\right) \in B$ such that $\mathbf{x}_{i} \in \mathbf{X}, n_{i}=\psi\left(\mathbf{x}_{i}\right), 1 \leq i \leq m$.

It is not hard to check that the projection of $\kappa_{m}$ to the first component $\partial \mathbb{D}^{m}$ of the Cartesian product $(\partial \mathbb{D})^{m} \times \mathbb{Z}_{+}^{m}$ is absolutely continuous with respect to $\mathrm{d} v\left(\mathbf{x}_{1}\right) \times \ldots \times$ $\mathrm{d} v\left(\mathbf{x}_{m}\right)$ where $v$ is the Riemannian volume on $\partial \mathbb{D}$. Taking the corresponding RadonNicodym derivative leads to a non-negative function

$$
\left(\left(\mathbf{x}_{1}, \ldots, \mathbf{x}_{m}\right),\left(n_{1}, \ldots, n_{m}\right)\right) \in(\partial \mathbb{D})^{m} \times \mathbb{Z}_{+}^{m} \mapsto \mathfrak{k}_{m}\left(\left(\mathbf{x}_{1}, n_{1}\right) \ldots,\left(\mathbf{x}_{m}, n_{m}\right)\right) .
$$

'Physically speaking', the sum

$$
\overline{\mathfrak{k}}_{m}\left(\mathbf{x}_{1}, \ldots, \mathbf{x}_{m}\right)=\sum_{n_{1}, \ldots, n_{m} \in \mathbb{Z}_{+}} \mathfrak{k}_{m}\left(\left(\mathbf{x}_{1}, n_{1}\right) \ldots,\left(\mathbf{x}_{m}, n_{m}\right)\right)
$$


describes the joint $m$-fold density of points $\mathbf{x} \in \mathbf{X}\left(\right.$ at $\left.\left(\mathbf{x}_{1}, \ldots, \mathbf{x}_{m}\right)\right)$, and the ratio

$$
\frac{\mathfrak{k}_{m}\left(\left(\mathbf{x}_{1}, n_{1}\right) \ldots,\left(\mathbf{x}_{m}, n_{m}\right)\right)}{\overline{\mathfrak{k}}_{m}\left(\mathbf{x}_{1}, \ldots, \mathbf{x}_{m}\right)}
$$

the 'conditional probability' of the event that $\psi\left(\mathbf{x}_{i}\right)=n_{i}, i=1, \ldots, m$, 'given' that points $\mathbf{x}_{1}, \ldots, \mathbf{x}_{m} \in \mathbf{X}$.

Theorem 10. As $\lambda \rightarrow \infty$,

(i) the measure $\lambda^{-n(n-1) /(n+1)} \kappa_{1}$ converges in variation to the measure $\kappa^{0}(d \mathbf{x})=$ $v(d \mathbf{x}) \sqrt{\mathbf{Q}(\mathbf{x})}$;

(ii) for $\forall m>1$, the measure $\lambda^{-m n(n-1) /(n+1)} \kappa_{m}$ converges in variation to $\left(\kappa^{0}\right)^{m}$, the Cartesian power of $\kappa^{0}$.

\section{ABOUT THE PROOF}

This section contains an outline of the proof of the results stated in the paper. The omitted technicalities are, as a rule, straightforward and do not contain any essential novelty.

Proof of Theorems 1, 2. The proof of these theorems is very similar. To verify the assertion of Theorem 1, it suffices to check that the integral over $\mathcal{M}^{0}$ is infinite. Here, $\mathcal{M}^{0}$ is the component of $\mathcal{M}$ where ground polyhedron $\mathbb{P}(\mathbf{Y})$ is an $(n-1)$-dimensional simplex (i.e., a convex polyhedron with $n$ vertices), and the integer distance from the origin to the ground face of veil $\mathcal{V}(\mathbf{Y})$ equals one. Measure $\nu^{0}(d \mathbf{Y})$ on $\mathcal{M}^{0}$ is proportional to

$$
\prod_{1 \leq j \leq n} d y_{j} /(V(\mathbf{Y}))^{n} .
$$

Here, $\mathbf{Y}_{j}\left(=y_{j}(\mathbf{Y})\right), j=1, \ldots, n$, are co-ordinates of $n$ vertices of simplex $\mathbb{P}(\mathbf{Y})$; each $\mathbf{Y}_{j}$ is an $(n-1)$-dimensional vector $\left(y_{j}^{1}, \ldots, y_{j}^{n-1}\right)$. Furthermore, $V(\mathbf{Y})$ is the $(n-1)$ dimensional volume of $\mathbb{P}(\mathbf{Y})$.

Pictorially, by changing $\mathbf{y}=\left(y_{j}, 1 \leq j \leq n\right)$, we vary the 'location' of the simplex, its 'shape' and a 'homothetic' factor $\ell>0$ (the latter is just a scaling factor). Both the location and shape variables run over compact domains; the only unrestricted quantity is $\ell$. It is convenient to perform a change of variables and separate variable $\ell$ from the rest. After such change, the LHS of (5.1) can be written as

$$
d \mathbf{y}_{\text {loc }} d \mathbf{y}_{\mathrm{sh}} d \ell J / V^{n}
$$

Here $\mathbf{y}_{\text {loc }}$ is the collection of variables responsible for the location of the simplex, and $\mathbf{y}_{\mathrm{sh}}$ is that determining its shape. Furthermore, $J=J\left(\mathbf{y}_{\text {loc }}, \mathbf{y}_{\mathrm{sh}}, \ell\right)$ is the Jacobian and $V=V\left(\mathbf{y}_{\text {loc }}, \mathbf{y}_{\mathrm{sh}}, \ell\right)$, as before, the volume of the simplex. 
The next key point of the proof of Theorem 1 is an observation, based on Minkovski's convex body theorem: the number of integer points inside a convex polyhedron grows proportionally to its (Euclidean) volume, where the proportionality coefficient is bounded below and above provided that the ratio of the radii of the inscribed and circumscribed spheres of the polyhedron (which in our case is a simplex) is kept within an interval $\left(\kappa_{0}, \kappa_{1}\right)$, where $\kappa_{0}, \kappa_{1}>0$ are fixed numbers. As the last condition extracts a subset of positive measure, we can replace, in the RHS of (2.5), quantity $N(\mathbf{Y})$ by volume $V(\mathbf{Y})$.

Thus we have to analyse the integrability, in variable $\ell>0$, of the expression

$$
d \ell \frac{J V}{V^{n}}=d \ell \frac{J}{V^{n-1}} .
$$

Now observe that the determinant $J \sim \ell^{(n-1)^{2}-1}$ (in fact, $(n-1)^{2}-1=n(n-1)-(n-1)-1$ is the number of scalar variables, among $\mathbf{Y}_{j}, 1 \leq j \leq n$, tied to $\ell$ ). On the other hand, $V^{n-1} \sim \ell^{(n-1)^{2}}$. Therefore (5.3) behaves like $d \ell / \ell$, which gives the divergence.

Remarks. (1) The expression $d \ell J / V^{n}$ gives a convergent integral: this reflects the fact that $\nu^{0}$ is a probability measure on $\mathcal{M}$.

(2) Observe that the order of divergence $d \ell / \ell$ emerging from the above argument does not depend on dimension $n$ (and is the same as for $n=2$ ).

Now let us turn to the proof of Theorem 2. The fact that the $\operatorname{limit} \lim _{R \rightarrow \infty} N_{R}^{1}(\mathbf{Y})$ $=\infty$ is a direct consequence of Theorem 1 and the ergodicity of flow $(\mathcal{E}, T, \nu)$. After this, to check that $\lim _{R \rightarrow \infty} N_{R}^{2}(\mathbf{Y})=\infty$, it suffices to check that $m_{R}(\mathbf{Y}) / v_{R}$ is separated from 0 with $\nu$-probability one as $R \rightarrow \infty$. This essentially means that a majority of the cells lying inside ball $\mathbb{B}_{R}$ are of a 'moderate' volume. A sufficient conditions is that the integral

$$
I(\mathbf{Y})=\sqrt{V_{\Delta}} \int_{\mathbb{P}(\mathbf{Y})} \frac{d y}{\prod_{1 \leq k \leq n} \operatorname{dist}\left(y, \partial_{k} \Delta\right)}
$$

is uniformly bounded below as a function of $\mathbf{Y} \in \mathcal{M}^{0}$, provided that the radii of the inscribed and circumscribed spheres of the ground polyhedron $\mathbb{P}(\mathbf{Y})$ (which is a simplex for $\mathbf{Y} \in \mathcal{M})$ are confined to a fixed interval $\left(R_{0}, R_{1}\right)$, with $R_{0}, R_{1}>0$. Here, $\Delta$ $(=\Delta(\mathbf{Y}))$ is the $(n-1)$-dimensional simplex obtained as the intersection of the translated hyperplane $\mathbb{G}(\mathbf{Y})$, when it reaches the ground face of $\mathcal{V}(\mathbf{Y})$, with the interior of the positive orthant (or the positive simplicial cone) generated by vectors $e_{1}(\mathbf{Y}), \ldots, e_{n}(\mathbf{Y})$. (Recall that $\mathbb{G}(\mathbf{Y})=\left\{x \in \mathbb{R}^{n}: x=\sum \mu_{i} e_{i}(\mathbf{Y})\right.$, where $\left.\sum \mu_{i}=0\right\}$.) Thus, $\Delta \supset \mathbb{P}(\mathbf{Y})$. As above, $V(\Delta)$ denotes the (Euclidean) volume of simplex $\Delta$. Furthermore, $\partial_{k} \Delta$ is an $(n-2)$-dimensional face of the boundary $\partial \Delta$ (symbol $k$ is simply a label for such a face; altogether there are $n$ of them). Finally, dist $\left(y, \partial_{k} \Delta\right)$ is the (Euclidean) distance from point $y \in \mathbb{P}(\mathbf{Y})$ to $\partial_{k} \Delta$.

The factor $\sqrt{V(\Delta)}$ is bounded from below by the square root of the volume of the $(n-1)$-dimensional ball of radius $R_{0}$. The volume of this ball also provides a lower bound for the volume of simplex $\mathbb{P}(\mathbf{Y})$. Thus, it remains to produce an upper bound 
for the product $\prod_{1 \leq k \leq n}$ dist $\left(y, \partial_{k} \Delta\right)$. We present a corresponding argument in the case $n=3$, where simplex $\Delta$ is a plane triangle (and so is $\mathbb{P}(\mathbf{Y})$ ). The modifications needed for the general case are purely technical and left to the reader.

Recall, that $\mathbb{P}(\mathbf{Y})$ is a triangle with integer vertices, say $z^{(1)}, z^{(2)}, z^{(3)}$, and that there should be no integer points in $\Delta \backslash \mathbb{P}(\mathbf{Y})$. Thus, $\forall i, i^{\prime}$, with $1 \leq i<i^{\prime} \leq 3$, there must be a side of $\mathbb{P}(\mathbf{Y})$ that crosses the segment with the endpoints $z^{(i)}-\left(z^{\left(i^{\prime}\right)}-z^{(i)}\right)$ $=2 z^{(i)}-z^{\left(i^{\prime}\right)}$. From this fact it is easy to deduce that two sides of triangle $\Delta$ cross a circle of radius $3 R_{1}$ which contains $\mathbb{P}(\mathbf{Y})$, and thus the distance from $\mathbf{Y} \in \mathbb{P}(\mathbf{Y})$ to any of them is $\leq 3 R_{1}$ (recall that $R_{1}$ is a bound for the radius of the circumscribed circle for $\mathbb{P}(\mathbf{Y}))$.

Now observe that each of the sides $\left(z^{\left(i^{\prime}\right)}-z^{(i)}\right)$ of ground triangle $\mathbb{P}(\mathbf{Y})$ forms, with any of the two aforementioned sides of $\Delta$, an angle that is $\geq \theta^{0}$ where $\theta^{0}\left(=\theta^{0}\left(R_{0}, R_{1}\right)\right)>$ 0 . Take one of the sides, say $z^{(1)}-z^{(2)}$, and draw, on the translated plane $\mathbb{G}(\mathbf{Y})+s$, the lines parallel $z^{(1)}-z^{(2)}$ through integer points. The distance between two such subsequent lines is $\leq 2 R_{1}$, and the spacing (i.e. the distance between two neighbour integer points) on each line is $\leq\left\|z^{(1)}-z^{(2)}\right\|$. It is then easy to see that the third side of $\Delta$ should be at distance $\leq R_{2}=R_{2}\left(R_{0}, R_{1}\right)$ from $y \in \mathbb{P}(\mathbf{Y})$. This leads to the desired bound for $\prod_{1 \leq k \leq 3} \operatorname{dist}\left(y, \partial_{k} \Delta\right)$.

Proof of Lemma 3.1. We will check that if $\widetilde{\delta}>0$ is small enough then for any two vertices $v, v^{\prime}$ of the veil the inequality $\operatorname{dist}_{\mathbb{R}^{n-1}}\left(\Pi v, \Pi v^{\prime}\right) \leq \delta$ is impossible. In fact, assume the opposite. Without loss of generality, we can think that one of the points $\Pi v$, $\Pi v^{\prime}$ is at the origin. Then, for the inverse images in $\mathbb{R}^{n}$, we have that $v$ is of the form $(\lambda, \ldots, \lambda)$ and $v^{\prime}$ of the form $\left(\lambda^{\prime}+\vartheta_{1}, \ldots, \lambda^{\prime}+\vartheta_{n}\right)$ where

$$
\left|\vartheta_{i}\right| \leq c \widetilde{\delta}, \text { and }\left|\frac{\lambda}{\lambda^{\prime}}-1\right| \leq c \widetilde{\delta}
$$

and $c$ is a positive constant (depending on dimension $n$ only). Then, for $\widetilde{\delta}$ small enough, $w=2 v-v^{\prime}$ is an integer point with positive co-ordinates, i.e. $w \in \mathcal{C}(\mathbf{Y})$, the Klein polyhedron. Thus, $v=\frac{1}{2}\left(w+v^{\prime}\right)$ cannot be a vertex.

Proof of Theorem 4. The quotient projections $G \rightarrow G_{0}=\operatorname{SL}(n, \mathbb{R})$ and $\Gamma \rightarrow$ $\Gamma_{0}=\mathrm{SL}(n, \mathbb{Z})$ determine a fibre bundle

$$
\pi: G / \Gamma \rightarrow G_{0} / \Gamma_{0}
$$

with the (natural) action of group $G$. The fibre $\pi^{-1}(x), x \in G_{0} / \Gamma_{0}$, of $\pi$ is a torus $\sim \mathbb{R}^{n} / \mathbb{Z}^{n}$. Thus, points $X \in G / \Gamma$ may be written as pairs $X=(x, \theta), x \in G_{0} / \Gamma_{0}$, 
$\theta \in \pi^{-1}(x)$. On the base $G_{0} / \Gamma_{0}$ we have a probability measure $\nu_{0}$ generated by the Haar measure on $\operatorname{SL}(n, \mathbb{R})$; measure $\nu$ is generated by $\nu_{0}$ and the (normalised) Lebesgue measures $\lambda_{x}$ on $\pi^{-1}(x)$. Let $U \subset G / \Gamma$ be a $T^{\prime}$-invariant set with $\nu(U)<1$ and $\chi_{U}$ its indicator function. We want to prove that $\nu(U)=0$. The action of group $G$ (and hence that of $T$ ) preserves the affine structure on tori; this enables us to define a function $F_{U} \geq 0$ on the dual fibre bundle

$$
\widetilde{\pi}: D \rightarrow G_{0} / \Gamma_{0}
$$

where the fibre $\tilde{\pi}^{-1}(x), x \in G_{0} / \Gamma_{0}$, is the first cohomology group $H^{1}\left(\pi^{-1}(x), \mathbb{Z}\right) \sim \mathbb{Z}^{n}$. Function $F_{U}$ is simply the square of the modulus of the Fourier transform of $\chi_{U}$ in the fibre variable $\theta$ (unlike the Fourier transform itself, its modulus is correctly defined). By Parcevale's identity, $F_{U}$ obeys $\sum_{\vartheta \in \tilde{\pi}^{-1}(x)} F_{U}(x, \vartheta) \leq 1$. Furthermore, there is a (natural) action of $G$ on $D$, through projection $G \rightarrow G_{0}=\mathrm{SL}(n, \mathbb{R})$, and hence an action of group $T^{\prime}$, through the induced projection $T^{\prime} \rightarrow T_{0}^{\prime}$ where $T_{0}^{\prime} \subseteq G_{0}$ is a non-compact subgroup, and $F_{U}$ is $T_{0}^{\prime}$-invariant.

It suffices to prove that the support $\operatorname{supp} F_{U}$ is not concentrated in the zero section $D_{(0)}=\left\{\left(x, \vartheta_{x}^{0}\right): x \in G_{0} / \Gamma_{0}\right\}$ of bundle $D$, with $\vartheta_{x}^{0}$ being the nil-cohomology class from $H^{1}\left(\pi^{-1}(x), \mathbb{Z}\right)$. In fact, if $\operatorname{supp} F_{U} \subseteq D_{(0)}$ then $\chi_{U}$ is $\lambda_{x}$-a.s. constant on $\pi^{-1}(x)$ for $\nu$-a.a. $x \in G_{0} / \Gamma_{0}$. Thus, $U$ is a union of fibres, modulo a set of $\nu$-measure 0 . That is, $\nu\left(U \triangle\left(\pi^{-1}\left(U_{0}\right)\right)\right)=0$ where $U_{0} \subseteq G_{0} / \Gamma_{0}$; set $U_{0}$ must have $\nu_{0}\left(U_{0}\right)>0$ and be invariant under the action of group $T_{0}^{\prime}$. Therefore, by Moore's ergodic theorem [M 1], [Z], $U_{0}=$ $G_{0} / \Gamma_{0}$ which implies that $\nu(U)=1$.

Write

$$
D \backslash D_{(0)}=\cup_{1 \leq k<\infty} D_{(k)},
$$

where

$$
\begin{array}{r}
D_{(k)}=\left\{(x, \vartheta): x \in G_{0} / \Gamma_{0}, \vartheta \in H^{1}\left(\pi^{-1}(x), \mathbb{Z}\right), \vartheta=k \vartheta_{0},\right. \\
\text { where } \left.\vartheta_{0}\left(=\vartheta_{0}(x)\right) \text { is a primitive element of } \mathbb{Z}^{d}\right\} .
\end{array}
$$

Note that $D_{(0)}, D_{(1)}, \ldots$, are connected components of $D$. Furthermore, it is easy to see that $D_{(0)} \sim G_{0} / \Gamma_{0}$. Finally, as we show below, $D_{(k)} \sim G_{0} / \Gamma_{1}, k=1,2 \ldots$, where $\Gamma_{1}$ is the subgroup of $\Gamma_{0}$ preserving the first co-ordinate in $\mathbb{Z}^{n}$.

In fact, observe that $D$ is $\sim$ to the set of pairs $(\Lambda, \gamma)$, where $\Lambda$ is a lattice in $\mathbb{R}^{n}$, with $0 \in \Lambda$ and $\operatorname{vol}\left(\mathbb{R}^{n} / \Lambda\right)=1$, and $\gamma$ is a homomorphism $\Lambda \rightarrow \mathbb{Z}$; in this language, $D_{(k)}$ $\sim\{(\Lambda, \gamma): \gamma(\Lambda)=k \mathbb{Z}\}$, and, clearly, $D_{(k)} \simeq D_{(1)}, k \geq 1$. So it is enough to check that $D_{(1)} \sim G_{0} / \Gamma_{1}$, which is clear from the fact that $G_{0} / \Gamma_{0}$ is $\sim$ the set of lattices $\Lambda \subset \mathbb{R}^{n}$ with $0 \in \Lambda$ and $\operatorname{vol}\left(\mathbb{R}^{n} / \Lambda\right)=1$ and hence $G_{0} / \Gamma_{1}$ itself is $\sim$ the set of pairs $(\Lambda, \gamma)$ where $\Lambda$ is as before and $\gamma$ is an epimorphism $\Lambda \rightarrow \mathbb{Z}$ (the set of such epimorphisms is denoted by $\operatorname{Ep}(\Lambda, \mathbb{Z}))$.

So, we assume that $F_{U} \Upsilon_{D_{(k)}} \neq 0$ for some $k \geq 1$. Hence we obtain a function on $G_{0} / \Gamma_{1}$ which we again denote by $F_{U}$ such that (i) $F_{U} \geq 0$ and $F_{U}>0$ on a set of positive measure, (ii) $\sum_{\gamma \in \operatorname{Ep}(\Lambda, \mathbb{Z})} F(\Lambda, \gamma) \leq 1$, (iii) $F_{U}$ is invariant under the action of a non-compact subgroup $T_{0}^{\prime} \subset G_{0}$. As follows from (i), (ii), $F_{U}$ is integrable on $G_{0} / \Gamma_{0}$. 
We will use the following geometric fact concerning the left quotient space $T_{0}^{\prime} \backslash G_{0}$ :

Lemma 5.1. The double coset $T_{0}^{\prime} \backslash G_{0} / \Gamma_{1}$ contains a subset $\mathcal{V}$ such that the inverse image $\mathcal{W}$ of $\mathcal{V}$, under the projection $T_{0}^{\prime} \backslash G_{0} \rightarrow T_{0}^{\prime} \backslash G_{0} / \Gamma_{1}$, has the following property. $\mathcal{W}$ is an open subset of full measure of the manifold $T_{0}^{\prime} \backslash G_{0}$, it is invariant under the right action of $\Gamma_{1}$, and $\Gamma_{1}$ acts on $\mathcal{W}$ discontinuously and freely. That is, $\forall v \in \mathcal{W}$ there exists an open neighbourhood $\mathcal{W}(v) \subseteq \mathcal{W}$ such that the $\operatorname{map}(\beta, u) \in \Gamma_{1} \times \mathcal{W}(v) \mapsto \beta(u) \in \mathcal{W}$ is an injection.

Proof of Lemma 5.1. It suffices to check the property stated in Lemma 1 for $T^{\prime}=T$. Observe that the image $T_{0}$ of group $T$ under projection $G \rightarrow G_{0}$ is the stabiliser of cylinder $\mathbb{V}_{0}=\left\{\left(x_{1}, \ldots, x_{n}\right) \in \mathbb{R}^{n}: \sum_{j=1}^{n-1} x_{j}^{2}=1\right\}$. Left quotient space $T_{0} \backslash G_{0}$ is the set of such cylinders $\mathbb{V}$, i.e., the set of non-negative quadratic forms $q_{\mathbb{V}}$ in $n$ variables of rank $n-1$. Together with the above description of right quotient space $G_{0} / \Gamma_{1}$, this leads to the following description of the double quotient space $T_{0} \backslash G_{0} / \Gamma_{1}$ : it is $\sim$ to the set of equivalence classes, under the action of $G_{0}$, of triples $\left(\Lambda, \gamma, q_{\mathbb{V}}\right)$. Here pair $(\Lambda, \gamma)$ is as in the case of $G_{0} / \Gamma_{1}$, and $q_{\mathbb{V}}$ is a quadratic form in $n$ variables of rank $n-1$ (i.e., a cylinder $\mathbb{V}$ in $\left.\mathbb{R}^{n}\right)$.

Given a representative $(\Lambda, \gamma, \mathbb{V})$ of a double coset from $T_{0} \backslash G_{0} / \Gamma_{1}$, consider the linear continuation $\widetilde{\gamma}(=\widetilde{\gamma}(\gamma))$ of map $\gamma$ from $\Lambda$ to the whole $\mathbb{R}^{n}$. Call the triple $(\Lambda, \gamma, \mathbb{V})$ good if linear form $\widetilde{\gamma}$ does not vanish along the axis of cylinder $\mathbb{V}$. The set $\widetilde{\mathcal{V}}$ of good triples forms an $\mathrm{SO}(n-1, \mathbb{R})$-orbifold, and in particular is a Hausdorff space. Namely, the nondegenerate quadratic form $\widetilde{q}=\widetilde{\gamma}^{2}+q_{\mathbb{V}}$ determines a Riemannian volume on $\mathbb{R}^{n}$; in some co-ordinates this volume has the form $\mathrm{d} x_{1} \cdots \mathrm{d} x_{n}$ whereas $\widetilde{q}\left(x_{1}, \ldots, x_{n}\right)=\sum_{j=1}^{n-1} x_{j}^{2}$ and $\widetilde{\gamma}\left(x_{1}, \ldots, x_{n}\right)=\eta x_{n}$ where $\eta>0$. As such co-ordinates are defined only up to the action of $\mathrm{SO}(n-1, \mathbb{R})$, this results in the structure of an orbifold, not a manifold, on $\widetilde{\mathcal{V}}$. However, after removal from $\widetilde{\mathcal{V}}$ of a suitable set of lesser dimension, the remaining set, $\mathcal{V}$, forms a manifold, and the action of $T_{0}$ on its inverse image $\mathcal{W}$ is discontinuous and free.

We continue with the proof of Theorem 4. Owing to Lemma 5.1, we can take the inverse image $V_{0}$ of $\mathcal{V}$ under the projection $G_{0} / \Gamma_{0} \rightarrow T_{0}^{\prime} \backslash G_{0} / \Gamma_{1}$. Set $V_{0}$ is again of full measure, and we can pass from $F_{U}$ to its restriction to $V_{0}$ for which we retain the previous notation $F_{U}$; clearly properties (i)-(iii) above hold true for the new $F_{U}$. Then $F_{U}$ must be integrable on $G_{0} / \Gamma_{1}$. However, this function arises from a function $F_{U}^{1}$ on $\mathcal{V}$; by Fubini's theorem, the integral of $F_{U}$ equals that of $F_{U}^{1}$ times the volume of group $T_{0}^{\prime}$. The latter is infinite as $T_{0}^{\prime}$ is non-compact. The contradiction obtained completes the proof of Theorem 4 .

Acknowledgements. We are grateful to V.I. Arnol'd for his encouragement and constant interest. We thank D. Anosov, M. Gordin, B. Gurevich, I. Ibragimov, A. Katok, S. Kerov, J. Lagarias, J. Lebowitz, G. Margulis, J. Marklof, N. Moshchevitin, M. Ratner, 
D. Ruelle, Ya. Sinai, A. Starkov and A. Vershik for their interest and useful remarks. MLK and YMS thank, respectively, DPMMS, University of Cambridge, and I.H.E.S., Bures-sur-Yvette, for hospitality during visits in 1997/8 and 1998/9. Special thanks go to S. Shea-Simonds for checking the style of the paper.

\section{REFERENCES}

[AF 1] R. Adler, L. Flatto. Cross section maps for geodesic flows, I. In: Ergodic Theory and Dynamical Systems. Proceedings. Special year, Maryland, 1979-1980 (A. Katok, Ed.). Progress in Mathematics, 21. Birkhäuser, Boston, 1982, pp. 103-161.

[AF 2] R. Adler, L. Flatto. The backward continued fraction map and geodesic flow. Ergodic Theor. Dyn. Systems, 4 (1984), 487-492.

[AF 3] R. Adler, L. Flatto. Geodesic flows, interval maps and symbolic dynamics. Bull. Amer. Math. Soc., 25 (1991), 229-334.

[Ar 1] V.I. Arnold. Statistics of integral convex polygons. Functional Anal. Appl., 14 (1980), no 2, 1-3.

[Ar 2] V.I. Arnold. A-graded algebras and continued fractions. Comm. Pure Appl. Math., 42 (1989), 993-1000.

[B] A. Borel. Linear algebraic groups. Berlin: Springer-Verlag, 1991.

[Br] A.J. Brentjes. Multi-Dimensional Continued Fraction Algorithms. Mathematical Centre Tracts, No 145. Amsterdam: Mathematical Centrum, 1981.

[C] J.W.A. Cassels. An Introduction to the geometry of numbers. Springer-Verlag, Berlin, 1959.

[CdV 1] Y. Colin de Verdière. Nombre de point entiers dans une famille homothétique de domaines de $\mathbf{R}^{n}$. Ann. Sci. École Norm. Sup., (4)10 (1977), 559-575.

[CdV 2] Y. Colin de Verdière. Nombre de point entiers dans une famille homothétique de domaines de $\mathbf{R}^{n}$. Séminaire Goulaouic-Schwartz (1076/1977). Équations aux dérivées partielles et analyse founctionnelle. Exp. No 23. École Polytech., Palaiseau, 1977.

[CFS] I.P. Cornfeld, S.V. Fomin and Ja.G. Sinai. Ergodic theory. Berlin: Springer-Verlag, 1982.

[DRS] W. Duke, Z. Rudnick and P. Sarnak. Density of integer points on affine homogeneous varieties. Duke Math. J., 71 (1993), 143-180. 
[GL] P.M. Gruber and C.F. Lekkerkerker. Geometry of numbers. North-Holland, Amsterdam, 1987.

[K] E. Korkina. Two-dimensional continued fractions. The simplest examples. Proc. Steklov Math. Inst., 209 (1995), 124-144.

[L] G. Lachaud. Voiles et polyèdres de Klein, 1,2. Preprints No 95-22. Laboratiore de Mathématiques Discrètes, CNRS, Marseiile-Luminy, 1995.

[La] J.C. Lagarias. Geodesic multidimensional continued fractions. Proc. London Math. Soc., (3)69 (1994), 464-488.

[M 1] G.A. Margulis. Discrete subgroups of semisimple Lie groups. Berlin: SpringerVerlag, 1991.

[M 2] G.A. Margulis. Dynamical and ergodic properties of subgroup actions on homogeneous spaces with applications to number theory. In: Proc. Int. Congress Math., vol. I (Kyoto, 1990). Math. Soc. Japan, Kyoto, 1991, pp. 193-215

[S] C. Series. The modular surface and continued fractions. Journ. London Math. Soc., 31 (1985), 69-80.

[Sk] M.M. Skriganov. Ergodic theory on $S L(n)$, Diophantine approximations and anomalities in the lattice point problem. Invent. Math., 132 (1998), 1-72.

[Z] R. Zimmer. Ergodic theory and semisimple groups. Boston: Birkhäuser, 1984. 